\title{
Mutual phenomena involving J5 Amalthea in 2002-2003
}

\author{
F. Vachier, J. E. Arlot, and W. Thuillot \\ Institut de mécanique céleste et de calcul des éphémerides, IMCCE-Observatoire de Paris \\ e-mail: arlot@bdl.fr; thuillot@bdl.fr \\ Received 30 July 2002 / Accepted 11 September 2002

\begin{abstract}
Every six years mutual eclipses and occultations occur among the Jovian system of satellites. Very accurate astrometric measurements and several physical characteristics of the surfaces can be infered from their observation. This paper is provide predictions of this type of events involving the fifth satellite J5 Amalthea, spanning from November 2002 to June 2003 and to urge astronomers to observe them. Only the predictions of the eclipses of Amalthea by Io are presented, when the distance between Amalthea-Io and Amalthea-Jutpiter is large enough for photometric purposes. A full list of phenomena is available on the server http://www.imcce.fr/Phemu03/phemu03_eng.html
\end{abstract}

Key words. Amalthea - mutual events - dynamical modeling

\section{Introduction}

The next passage of the Earth and Sun through the Jovian equatorial plane will be the opportunity to observe mutual events when the Jovian satellites will eclipse and occult each other starting from the end of the year 2002. Usually, and since 1973, mutual event observations concern the Galilean moons since their photometric observation is easy to perform. The Amalthea case is very different because of the faintness of this moon, its magnitude at mean opposition being close to $V=14$ (Nicholson 1991; Millis 1978). Furthermore, the semimajor axis of its orbit being 2.5 Jovian radii, this satellite moves close to Jupiter which will have a mean visible magnitude $V=-2.6$ at opposition in February 2003. Nevertheless several methods, such as the observation in infrared bands or using coronographic methods, can give access to these photometric observations in some high altitude locations. Relative photometry is only necessary in order to acquire lightcurves from which positional circumstances and scaterring light parameters can be deduced. Aksnes \& Franklin (1976) have previously recommended the observation of this type of event, but to our knowledge, no lightcurves could be obtained at this time. We hope that the improvement of detectors, and especially, the advent of CCD photometry and infrared observations will now allow success in these observations. One of our main goals is to obtain strong astrometric constraints for our dynamical model of Amalthea. Subsequently, this will allow us to improve the dynamical model of the three innermost satellites, whose motions are much less known, since their astrometric positions are generally relative to Amalthea.

Send offprint requests to: F. Vachier, e-mail: vachier@bdl . fr

\section{The dynamical model}

We have recently developed a new numerical model of the five inner faint satellites of Jupiter, J5 Amalthea, J14 Thebe, J15 Adrastea, J16 Metis, and have used this to predict the phenomena. This model is founded on the Bulirsch et al. (1966) algorithm, including different effects such as the oblateness of the planet and the gravitational effects of the Galilean satellites. This model has been fitted to more than 87 positional observations for Amalthea spanning 1988 to 2000. The accuracy of the model has thus been estimated at 200 mas (i.e. $600 \mathrm{~km}$ at mean opposition). Compared to JPL ephemeris, our model has a standard deviation of 120 mas on the same period of time. Further information will be published later (Vachier, in preparation). The mutual events presented here involve J5 Amalthea and the first Galilean satellite Io. The model of motion of Io is issued from the Sampson-Lieske theory (1977) of the Galilean satellites, adjusted by Arlot and labeled G-5 (1982).

\section{The predictions}

We computed eclipses of J5 Amalthea by the Galileans moons from November 2002 to June 2003, since these events could give access to the best signal/noise ratio that geometric circumstances would allow, for an apparent distance of the eclipsing satellite which was large enough to measure photometric variation of Amalthea alone. The signal to detect during occultations, when both satellites are measured, is too faint. We iteratively calculated the first and last contacts of the eclipsed satellite with the umbra cone (when an eclipse is detected) and its minimum distance to the axis of this cone. The relative positions of the cone and the satellites are calculated by taking 
Table 1. Mutual events between Amalthea and Io during the 2002-2003 period.

\begin{tabular}{|c|c|c|c|c|c|c|c|c|c|}
\hline $\begin{array}{c}\text { Julian Day } \\
\text { UT }\end{array}$ & $\begin{array}{c}\text { Date Greg. } \\
\text { UT }\end{array}$ & $\begin{array}{r}\Delta X^{a} \\
\operatorname{arcsec}\end{array}$ & $\begin{array}{r}\Delta Y^{b} \\
\operatorname{arcsec}\end{array}$ & $\begin{array}{c}\Delta_{\mathrm{Io}} \\
\operatorname{arcsec}\end{array}$ & $\begin{array}{c}\Delta_{\mathrm{J}} \\
\operatorname{arcsec}\end{array}$ & $\begin{array}{c}\rho \\
\operatorname{arcsec}\end{array}$ & $\begin{array}{c}\mathrm{d} t \\
\text { second }\end{array}$ & $\begin{array}{c}\mathrm{d} m \\
\operatorname{arcsec}\end{array}$ & $\begin{array}{c}\mathrm{d} p \\
\operatorname{arcsec}\end{array}$ \\
\hline 2452595.672984954 & 2002/11/17 04:09:05.89 & -18.494 & 6.756 & 19.68 & 48.94 & 18.99 & 0. & 0.76 & 0.57 \\
\hline 2452627.498471065 & $2002 / 12 / 18$ 23:57:47.90 & -10.767 & 3.678 & 11.37 & 36.45 & 20.85 & 1450. & 0.40 & 0.55 \\
\hline 2452630.844881944 & 2002/12/22 08:16:37.79 & -10.952 & 3.914 & 11.63 & 46.93 & 21.03 & 487. & 0.54 & 0.55 \\
\hline 2452631.060819444 & $2002 / 12 / 22$ 13:27:34.79 & -16.678 & 6.130 & 17.76 & 54.20 & 21.04 & 0. & 0.81 & 0.58 \\
\hline 2452646.991589121 & 2003/01/07 11:47:53.30 & -10.754 & 4.110 & 11.51 & 56.41 & 21.77 & 0. & 0.80 & 0.57 \\
\hline 2452655.634476852 & 2003/01/16 03:13:38.79 & -10.602 & 3.870 & 11.28 & 35.09 & 22.05 & 0. & 0.81 & 0.60 \\
\hline 2452687.507636574 & 2003/02/17 00:10:59.80 & 8.383 & -2.678 & 8.80 & 32.50 & 22.09 & 113. & 0.19 & 0.61 \\
\hline 2452691.019006944 & 2003/02/20 12:27:22.19 & 9.608 & -3.100 & 10.09 & 45.17 & 21.99 & 136. & 0.07 & 0.60 \\
\hline 2452694.535236111 & 2003/02/24 00:50:44.39 & 10.044 & -3.195 & 10.54 & 54.47 & 21.88 & 181. & 0.02 & 0.58 \\
\hline 2452698.070984954 & 2003/02/27 13:42:13.09 & 9.063 & -2.700 & 9.45 & 53.46 & 21.76 & 465. & 0.10 & 0.56 \\
\hline 2452698.314288194 & $2003 / 02 / 27$ 19:32:34.49 & 10.902 & -2.873 & 11.27 & 54.63 & 21.75 & 152. & 0.40 & 0.57 \\
\hline 2452701.834429398 & 2003/03/03 08:01:34.70 & 14.239 & -4.023 & 14.79 & 43.92 & 21.60 & 134. & 0.25 & 0.59 \\
\hline 2452706.955986111 & 2003/03/08 10:56:37.19 & 17.460 & -5.213 & 18.22 & 42.34 & 21.37 & 131. & 0.01 & 0.60 \\
\hline 2452710.470355324 & 2003/03/11 23:17:18.69 & 16.782 & -4.880 & 17.47 & 51.50 & 21.20 & 163. & 0.03 & 0.59 \\
\hline 2452713.995875000 & 2003/03/15 11:54:03.60 & 14.672 & -4.056 & 15.22 & 54.03 & 21.02 & 272. & 0.15 & 0.57 \\
\hline 2452714.245583334 & $2003 / 03 / 15$ 17:53:38.40 & 14.709 & -4.107 & 15.27 & 54.10 & 21.01 & 272. & 0.10 & 0.57 \\
\hline 2452717.770649306 & 2003/03/19 06:29:44.09 & 18.841 & -5.448 & 19.61 & 44.39 & 20.81 & 161. & 0.06 & 0.59 \\
\hline 2452722.893640046 & 2003/03/24 09:26:50.50 & 23.133 & -6.541 & 24.04 & 38.82 & 20.53 & 126. & 0.01 & 0.60 \\
\hline 2452726.406620370 & 2003/03/27 21:45:31.99 & 21.646 & -5.966 & 22.45 & 47.86 & 20.32 & 149. & 0.08 & 0.59 \\
\hline 2452729.927077546 & 2003/03/31 10:14:59.49 & 18.836 & -5.015 & 19.49 & 52.08 & 20.11 & 208. & 0.19 & 0.57 \\
\hline 2452730.171966435 & 2003/03/31 16:07:37.90 & 15.725 & -4.523 & 16.36 & 52.15 & 20.10 & 419. & 0.14 & 0.56 \\
\hline 2452737.222520833 & 2003/04/07 17:20:25.80 & 24.083 & -6.968 & 25.07 & 32.45 & 19.68 & 116. & 0.31 & 0.60 \\
\hline 2452738.831780093 & 2003/04/09 07:57:45.80 & 26.264 & -7.211 & 27.23 & 34.83 & 19.58 & 122. & 0.01 & 0.61 \\
\hline 2452742.343684028 & 2003/04/12 20:14:54.30 & 24.301 & -6.563 & 25.17 & 43.84 & 19.37 & 139. & 0.09 & 0.59 \\
\hline 2452745.860995370 & $2003 / 04 / 16$ 08:39:50.00 & 21.179 & -5.635 & 21.91 & 49.17 & 19.16 & 182. & 0.15 & 0.58 \\
\hline 2452749.406243056 & 2003/04/19 21:44:59.40 & 15.543 & -4.125 & 16.08 & 40.68 & 18.95 & 841. & 0.12 & 0.55 \\
\hline 2452749.641084491 & 2003/04/20 03:23:09.70 & 20.300 & -5.963 & 21.15 & 45.08 & 18.93 & 149. & 0.38 & 0.58 \\
\hline 2452753.159864584 & $2003 / 04 / 23$ 15:50:12.30 & 23.741 & -6.859 & 24.71 & 33.83 & 18.73 & 117. & 0.34 & 0.59 \\
\hline 2452754.770166667 & 2003/04/25 06:29:02.40 & 27.027 & -7.419 & 28.02 & 30.57 & 18.63 & 118. & 0.00 & 0.61 \\
\hline 2452758.281230324 & 2003/04/28 18:44:58.29 & 24.877 & -6.813 & 25.79 & 39.62 & 18.43 & 134. & 0.02 & 0.60 \\
\hline 2452761.796299769 & 2003/05/02 07:06:40.30 & 21.722 & -5.980 & 22.53 & 45.80 & 18.24 & 169. & 0.02 & 0.58 \\
\hline 2452765.324815972 & 2003/05/05 19:47:44.10 & 17.157 & -4.839 & 17.82 & 45.21 & 18.05 & 323. & 0.08 & 0.56 \\
\hline 2452765.574181713 & 2003/05/06 01:46:49.30 & 18.321 & -5.445 & 19.11 & 45.16 & 18.03 & 180. & 0.38 & 0.57 \\
\hline 2452769.096738426 & $2003 / 05 / 09$ 14:19:18.20 & 21.723 & -6.323 & 22.62 & 35.37 & 17.85 & 133. & 0.29 & 0.59 \\
\hline 2452774.219069445 & 2003/05/14 17:15:27.60 & 23.718 & -6.807 & 24.67 & 35.32 & 17.58 & 122. & 0.17 & 0.60 \\
\hline 2452777.732592592 & 2003/05/18 05:34:55.99 & 20.792 & -6.106 & 21.67 & 42.21 & 17.41 & 138. & 0.25 & 0.59 \\
\hline 2452781.254724537 & $2003 / 05 / 21$ 18:06:48.19 & 16.874 & -5.155 & 17.64 & 44.70 & 17.24 & 176. & 0.38 & 0.57 \\
\hline 2452781.503969907 & 2003/05/22 00:05:42.99 & 15.302 & -4.628 & 15.98 & 44.58 & 17.23 & 276. & 0.31 & 0.56 \\
\hline 2452785.033035879 & 2003/05/25 12:47:34.29 & 18.682 & -5.549 & 19.48 & 36.93 & 17.07 & 153. & 0.25 & 0.58 \\
\hline 2452790.157081019 & 2003/05/30 15:46:11.80 & 21.271 & -6.580 & 22.26 & 30.99 & 16.85 & 72. & 0.48 & 0.60 \\
\hline 2452793.669351852 & 2003/06/03 04:03:52.00 & 18.646 & -5.974 & 19.57 & 38.47 & 16.70 & 0. & 0.60 & 0.59 \\
\hline 2452797.187765046 & 2003/06/06 16:30:22.90 & 15.360 & -5.149 & 16.20 & 42.83 & 16.56 & 0. & 0.70 & 0.57 \\
\hline 2452797.385594907 & 2003/06/06 21:15:15.39 & 9.919 & -3.204 & 10.42 & 31.37 & 16.56 & 836. & 0.33 & 0.54 \\
\hline 2452800.968388889 & 2003/06/10 11:14:28.80 & 15.077 & -4.654 & 15.77 & 38.40 & 16.42 & 170. & 0.25 & 0.58 \\
\hline 2452804.485777778 & 2003/06/13 23:39:31.19 & 16.651 & -5.267 & 17.46 & 29.05 & 16.30 & 106. & 0.38 & 0.59 \\
\hline
\end{tabular}

a $\Delta X=\Delta \alpha \cos (\delta)$ is Differential coordinate of Amalthea relative to Io.

${ }^{b} \Delta Y=\Delta \delta$ is Differential coordinate of Amalthea relative to Io.

into account the aberration effects and the date of prediction includes the light-time delay.

A large set of predictions has been obtained and will be made available on the web server that we have set up, dedicated to the international campaign PHEMU03 for the observation of the Galilean satellites' mutual events (http: //www . bdl . fr/Phemu03/phemu03_eng.html).
In this paper we only give a selection of the most interesting phenomena, i.e. only the eclipses of J5 Amalthea by J1 Io, when elongation to Jupiter is large enough.

This is why these predictions are given from November 17, 2002 to June 13, 2003, when the elongation of Jupiter to the Sun is greater than 50 degrees. During this period, all events are observable all around the world depending on the date of 
the event and the local circumstances of elevation above the horizon.

We give in Table 1:

- the dates of the events in Julian day UT (Col. 1);

- and in Gregorian date UT (Col. 2);

- $\Delta X$ and $\Delta Y$, the differential coordinates of the satellite with respect to the center of Io (Cols. 3 and 4);

- $\Delta_{\text {Io }}$, the apparent distance from Io (Col. 5);

- $\Delta_{\mathrm{J}}$, the apparent distance from Jupiter (Col. 6);

- $\rho$, the apparent radius of Jupiter (Col. 7);

- $\mathrm{d} t$, the duration of the event in seconds of time, this value is 0 . when it concerns close approaches and grazing phenomena (Col. 8);

- $\mathrm{d} m$, the minimal apparent distance from the center of the umbra cone (Col. 9);

- $\mathrm{d} p$, the minimal apparent distance for an event to take place (Col. 10) which corresponds to the sum of the apparent radius of the satellite and to the apparent radius of the section of the umbra cone.

According to the accuracy of our model and considering a mean radius of $\mathrm{J} 5$ Amalthea $(120 \mathrm{~km})$ instead of a triaxial model (125 km, $73 \mathrm{~km}, 64 \mathrm{~km}$ ) (Thomas 1998), we include in this table only the phenomena or the grazing phenomena with $\mathrm{d} m-\mathrm{d} p$ smaller than 0.23 arcsec.

\section{Observing events}

The aim of these observations is to provide lightcurves of the satellite Amalthea when it crosses the umbra cone of Io, related to an accurate timing in Universal time. It is very desirable to obtain large enough data samples of data during the immersion and during the emersion from the umbra cone.

When the best CCD observations give 100 mas precision, it is interesting to give us a time base to obtain more precise observations. That is why, to efficiently observe these phenomena, we need to have an instrument powerful enough to obtain images with exposure and readout times with a minimal sampling of time that corresponds to 10 mas of accuracy in the orbit. According to Amalthea's radius $(120 \mathrm{~km})$, and calling $v$ its tangential velocity when it enters in the umbra cone, and $d$ the distance of the satellite from the Earth, we calculated the sampling of time $e$ with:

$e=\frac{d}{v}$
For the December, 18, 2002 event, $1.4 \mathrm{~s}$ of time would be necessary since this corresponds to $38.4 \mathrm{~km}$ (10 mas) in the orbit accuracy, according to a tangential velocity of Amalthea of $26.49 \mathrm{~km} \mathrm{~s}^{-1}$. These satellite configurations (Table 1) vary around this value of $e$ and depend if the satellite is near its opposition. This is why we suggest to observers to acquire $1 \mathrm{im}$ ages per second when Amalthea's flux is high enough. In this case, we have a precision on the position of the satellite, when it crosses the umbra cone of 6.89 mas. Of course, observations with a stronger time base remain interesting compared to CCD observations, and for one image every $7.25 \mathrm{~s}$ the precision is 50 mas. The best case is to be able to acquire one image every $0.14 \mathrm{~s}$ and the precision will be 1 mas.

\section{Conclusions}

During the periods of mutual eclipses and occultations of the Jovian satellites, which occur every six years, several mutual events involve the small inner satellites with a Galilean satellite. Due to the difficulty of observing such an event, no data have been obtained until now during previous campaigns. However, photometric observations of such events could give access to incomparably accurate astrometric measurements, useful for the dynamical modeling of these faint satellites. In this paper, we give a list concerning the most observable events of J5 Amalthea eclipsed by Io over the period November 2002 to June 2003. We hope that astronomers interested in these problems will join the international campaign that we have organized in order to succeed in these observations.

Acknowledgements. Thanks to the members of the GAP team of the Institut de Mécanique Céleste et de Calcul des Ephémérides (Paris) for their constructive discussions.

\section{References}

Aksnes, K., \& Franklin, F. A. 1978, Icarus, 34, 188

Aksnes, K., \& Franklin, F. A. 1976, AJ, 81, 464

Arlot, J.-E. 1982, A\&A, 107, 305

Arlot, J.-E. 2002, A\&A, 383, 719

Bulirsch, R., \& Stoer, J. 1966, Num. Math., 8, 1

Lieske, J. H. 1977, A\&A, 56, 333

Millis, R. L. 1978, Icarus, 33, 319

Nicholson, P. D., \& Matthews, K. 1991, Icarus, 93, 331

Stoer, J., \& Bulirsch, R. 1992, Introduction to numerical analysis (Springer-Verlag, New York)

Thomas, P. C., Burns, J. A., Rossier, L., et al. 1998, Icarus, 135, 360 